\title{
Research on Outage Performance Optimization of Cognitive Relay Network with Cooperative Sensing
}

\author{
Yaolian Song, YuHao Li, Shujuan Wang and Ya Zhang \\ Faculty of Information Engineering and Automation, Kunming University of Science and Technology, Yunnan, China
}

\begin{abstract}
In this work, cooperative spectrum sensing technology is applied to optimize the outage probability of cognitive relay networks. The outage performance optimization problem of cognitive relay networks is resolved by designing the sensing time and fusion rule threshold appropriately. The outage probability by high signal to noise ratio (SNR) approximation under Rayleigh fading channels is derived theoretically, and the solution of optimal fusion rule threshold is given. Theoretical analysis and simulation results illustrate that the optimal sensing time and fusion rule threshold exist to minimize the outage probability. The outage performance of cognitive relay networks is significantly improved under the assistance of cooperative sensing compared with that of non-cooperative sensing scheme.
\end{abstract}

ACM CCS (2012) Classification: Networks $\rightarrow$ Network components $\rightarrow$ Wireless access points, base stations and infrastructure $\rightarrow$ Cognitive radios;

Networks $\rightarrow$ Network performance evaluation $\rightarrow$ Network performance analysis

Keywords: outage probability, fusion rule threshold, SNR, sensing time

\section{Introduction}

Cognitive radio technology is the key technology to realize dynamic spectrum access, which can effectively solve the problem of spectrum resources shortage, and can provide precious spectrum resources to support the fast development of wireless communication. The detection performance of spectrum sensing can be drastically degraded, due to multipath fading, shadowing and receiver uncertainty. Therefore, cooperative spectrum sensing is considered to enhance sensing performance [1-3]. In [2,4], it is shown that the sensing time can be shorter and the flexibility of sensing can be improved by considering cooperative sensing. The throughput and spectrum efficiency of cognitive radio networks can be greatly improved by applying cooperative sensing based on hard decision fusion rule $[5,6]$, and the detection performance of hard decision fusion rule is approximate to that of soft decision fusion scheme.

In cognitive radio networks, the spectrum holes detection is related to data transmission. Therefore, they should be designed and optimized together. Spectrum sensing and data transmission were jointly designed in [7], and there existed optimal sensing time to maximize the throughput of cognitive radio networks under the constraint of enough protection of primary user. Cluster-based cooperative sensing was researched in [8]; the throughput of cognitive radio networks was significantly improved through sensing time and throughput tradeoff. The outage probability of cognitive relay networks was investigated in [9], the optimal sensing overhead was designed to minimize the outage probability of cognitive relay networks. In [10], authors investigated the outage performance of cognitive radio network where secondary receiver does not suffer any interference from primary user. The outage performance of cognitive relay networks under imperfect channel knowledge estimations was studied [11], and the impact of the number of relays and channel state information on the outage performance was analyzed. In [12], authors studied the outage performance of cognitive relay network based on decode and forward mode, and the results showed that both the relay selection scheme and the number of relays had great impact on the outage performance. In 
[13], the outage performance of cognitive relay networks was researched, and it was improved under relay-assisted transmission. In [14], authors investigated the transmission capacity of cognitive radio network under the outage constraints from the primary network with or without cooperative relaying, and analyzed the impact of the location of the relay.

However, the previous work did not take cooperative sensing into account to investigate the outage performance of cognitive relay network. In view of this, we investigate outage probability optimization problem of cognitive relay network based on cooperative sensing, in which cognitive users can periodically monitor the licensed frequency band to decide whether to transmit data or wait for the next frame. We establish a cognitive relay network model described by two variables, namely, sensing durations and fusion rule threshold. It is approved that there exist optimal sensing duration and an optimal fusion rule threshold to improve the outage performance of cognitive relay network.

The remainder of this paper is organized as follows: Section II depicts the system model. In Section III, the outage probability optimization of cognitive relay network is derived and explained in detail. In Section IV, the numerical and simulation results are demonstrated. Finally, Section V concludes this paper.

\section{System Model}

We consider a cognitive relay cooperative transmission system model as shown in Figure 1 , where the cognitive relay (CR) helps the cognitive source (CS) for data transmission. Each transmission link between any two nodes is modeled as a Rayleigh fading channel. One can see that the whole cognitive transmission process can be divided into two phases, namely the spectrum holes detection of the licensed spectrum band and the cognitive data transmis-

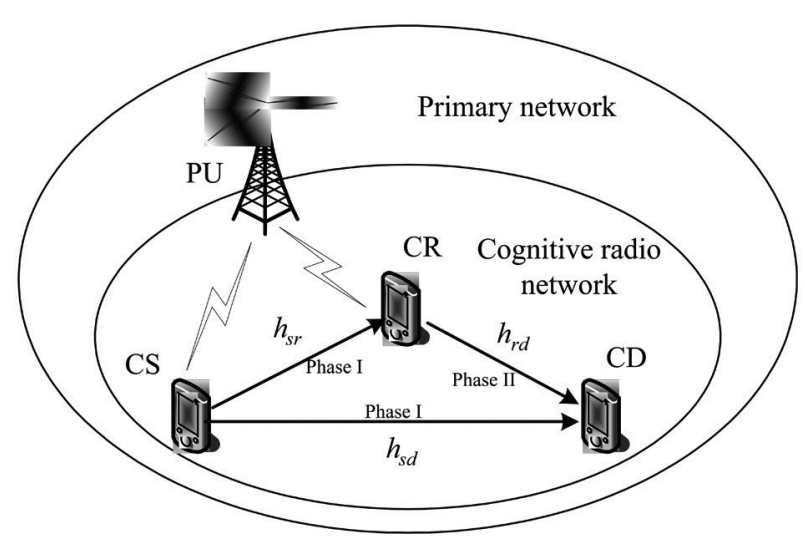

Figure 1. System model.

sion. As Figure 2 depicts, the cognitive data transmission phase is divided into one sensing slot and two data transmission slots. Suppose $\tau$ is the spectrum sensing duration and $T$ is the frame duration, then the overall data transmission duration is derived as $T-\tau$.

In cognitive relay networks, once cognitive users have detected idle licensed spectrum bands, they will utilize the chances to transmit data to their destinations in the assistance of cognitive relay. Each cognitive node is equipped with single antenna and cannot send and receive data at the same time. The cognitive relay helps the cognitive source forwarding information to the cognitive destination with amplify-and-forward (AF) mode. The cognitive relay cooperative transmission scheme is shown in Table 1.

Table 1. CR cooperative transmission scheme

\begin{tabular}{|c|c|}
\hline Slots & CR cooperative transmission scheme \\
\hline \hline Slot 1 & $\mathrm{CS} \rightarrow \mathrm{CR}, \mathrm{CD}$ \\
\hline Slot 2 & $\mathrm{CR} \rightarrow \mathrm{CD}$ \\
\hline
\end{tabular}

At the beginning of the cognitive data transmission phase, the cognitive source broadcasts its information to both the cognitive relay and the cognitive destination within the transmission slot 1. After that, CR retransmits the received

\begin{tabular}{|c|c|c|c|c|c|c|}
\hline $\begin{array}{c}\text { Spectrum } \\
\text { sensing }\end{array}$ & $\begin{array}{c}\text { CS transmit } \\
\text { data }\end{array}$ & $\begin{array}{c}\text { CR forward } \\
\text { data }\end{array}$ & $\begin{array}{c}\text { Spectrum } \\
\text { sensing }\end{array}$ & $\begin{array}{c}\text { CS transmit } \\
\text { data }\end{array}$ & $\begin{array}{c}\text { CR forward } \\
\text { data }\end{array}$ & $\ldots . .$. \\
\hline \\
\hline
\end{tabular}

Figure 2. Frame structures for cognitive transmission. 
data to $\mathrm{CD}$ with amplify-and-forward (AF) mode, while CS does not broadcast any information in the transmission slot 2. Throughout the paper, we assume that the channel coefficients are known by the receivers, but not by the transmitters, and there exists a perfect synchronization between cooperative nodes. Finally, the receiver combines multiple signals with maximal ratio combination. The normalized capacity of cognitive relay network can be expressed as [15].

$$
\begin{aligned}
I_{I}=\frac{T-\tau}{2 T} & \log _{2}\left(1+\frac{P_{s}\left|h_{s d}\right|^{2}}{\sigma_{n}^{2}}\right. \\
& \left.+\frac{P_{s} P_{r}\left|h_{s r}\right|^{2}\left|h_{r d}\right|^{2}}{\left(P_{s}\left|h_{s r}\right|^{2}+P_{r}\left|h_{r d}\right|^{2}+\sigma_{n}^{2}\right) \sigma_{n}^{2}}\right)
\end{aligned}
$$

where $P_{s}$ is the transmission power of the cognitive source and $P_{r}$ is the transmission power of the cognitive relay. We assume $P_{s}=P_{r}$ and $P_{s}+P_{r}=P$, is the allowable total power, $\sigma_{n}^{2}$ is the variance of Gaussian noise. $h_{s r}, h_{s d}$ and $h_{r d}$ denote the fading channel coefficients between $\mathrm{CS}$ and $\mathrm{CR}, \mathrm{CS}$ and $\mathrm{CD}, \mathrm{CR}$ and $\mathrm{CD}$, respectively. Each transmission link between any two nodes is modeled as a Rayleigh fading process with variances $\delta_{s d}^{2}, \delta_{s r}^{2}$ and $\delta_{r d}^{2}$.

\section{Outage Probability of Cognitive Relay Networks}

The outage probability of cognitive relay network is investigated in this section. In non-cognitive relay networks, the outage event will occur when channel capacity $I$ falls below $R$. Therefore, the outage probability is defined as $\mathrm{P}_{\text {out }}=\operatorname{Pr}(I<R)$, where $R$ is the required data transmission rate for the source. However, in cognitive relay networks, it is important to consider the activity of the primary user and the detection error at the cognitive user. In this paper, we modify the above definition of outage probability, in which the outage occurs when the following three cases meet:

Case 1: When the primary user is inactive and the cognitive source makes a correct decision, the outage event occurs if $I<R$.
Case 2: When the primary user is inactive, but the cognitive source makes a wrong decision and remains silent.

Case 3: When the primary user is active, but the cognitive source loses it. In this case, cognitive source continues transmitting data, the outage event happens when $I<R$.

It is obvious that the above three cases do not include the case that the primary user is active, and the cognitive source makes a correct decision and remains silent, which we cannot call as outage because it is a proper usage of the spectrum.

Using the total probability law, the outage probability of cognitive relay network can be obtained as follows:

$$
\begin{aligned}
& \mathrm{P}_{\text {out }}(\tau, k, \eta)=P\left(H_{0}\right)\left(1-\mathrm{P}_{F}(\tau, k, \eta)\right) \\
& \cdot \operatorname{Pr}\left(I<R \mid \hat{H}_{0}, H_{0}\right)+\left(1-\mathrm{P}_{D}(\tau, k, \eta)\right) P\left(H_{1}\right) \\
& \cdot \operatorname{Pr}\left(I<R \mid \hat{H}_{0}, H_{1}\right)+\mathrm{P}_{F}(\tau, k, \eta) P\left(H_{0}\right)
\end{aligned}
$$

Let $\hat{H}_{i}, i \in\{0,1\}$ denote the decision result of energy detection at the cognitive source, where $\hat{H}_{0}$ and $\hat{H}_{1}$ correspond to the decision that primary user is inactive and active respectively. $\tau$ is the spectrum sensing time, $k$ denotes the fusion rule threshold and meets with $1 \leq k \leq M$. $M$ is the number of cognitive users to participate in cooperative spectrum sensing. $\eta$ stands for the decision threshold of energy detector.

Let $P_{s}=P_{r}=P, \quad \gamma=\frac{P}{\sigma_{n}^{2}}$ denotes signal to noise ratio, $\operatorname{Pr}\left(I<R \mid \hat{H}_{0}, H_{0}\right)$ can be formulated as follows:

$$
\begin{aligned}
& \operatorname{Pr}\left(I<R \mid \hat{H}_{0}, H_{0}\right)=\operatorname{Pr}\left(I_{I}<R\right) \\
& =\operatorname{Pr}\left[\left|h_{s d}\right|^{2}+\frac{\gamma\left|h_{s r}\right|^{2}\left|h_{r d}\right|^{2}}{\gamma\left|h_{s r}\right|^{2}+\gamma\left|h_{r d}\right|^{2}+1}<\frac{2^{\frac{2 T R}{(T-\tau)}}-1}{\gamma}\right]
\end{aligned}
$$

where $\left|h_{s d}\right|^{2},\left|h_{s r}\right|^{2}$ and $\left|h_{r d}\right|^{2}$ are exponentially distributed with parameter $\frac{1}{\delta_{s d}^{2}}, \frac{1}{\delta_{s r}^{2}}$ and $\frac{1}{\delta_{r d}^{2}}$ respectively. It is difficult to obtain the exact outage probability. However, $\operatorname{Pr}\left(I<R \mid \hat{H}_{0}, H_{0}\right)$ 
can be obtained based on the outage probability of cooperative communication by high signal to noise ratio approximation [16].

$$
\begin{aligned}
\operatorname{Pr}(I & \left.<R \mid \hat{H}_{0}, H_{0}\right) \\
& =\frac{1}{2 \delta_{s d}^{2}}\left(\frac{1}{\delta_{s r}^{2}}+\frac{1}{\delta_{r d}^{2}}\right)\left(\frac{2^{\frac{2 T R}{(T-\tau)}}-1}{\gamma}\right)^{2}
\end{aligned}
$$

The received information of cognitive destination includes signal, noise, as well as interference from the primary user due to the sensing error of cognitive source in case 3 . Let signal interference ratio be $\gamma^{\prime}=\frac{P}{\sigma_{n}^{2}+P_{p}}=\frac{\gamma}{1+\gamma_{p}}$, then $\operatorname{Pr}\left(I<R \mid \hat{H}_{0}, H_{1}\right)$ can be written as follows:

$$
\begin{aligned}
& \operatorname{Pr}\left(I<R \mid \hat{H}_{0}, H_{1}\right) \\
& =\frac{1}{2 \delta_{s d}^{2}}\left(\frac{1}{\delta_{s r}^{2}}+\frac{1}{\delta_{r d}^{2}}\right)\left(\frac{\left(2^{\frac{2 T R}{(T-\tau)}}-1\right)\left(1+\gamma_{p}\right)}{\gamma}\right)^{2}
\end{aligned}
$$

The total outage probability of cognitive relay network can be expressed as

$$
\begin{aligned}
\mathrm{P}_{\text {out }}(\tau, k, \eta)=\mathrm{P}_{F}(\tau, k, \eta) P\left(H_{0}\right) \\
+P\left(H_{0}\right)\left(1-\mathrm{P}_{F}(\tau, k, \eta)\right) \frac{1}{2 \delta_{s d}^{2}} \\
\cdot\left(\frac{1}{\delta_{s r}^{2}}+\frac{1}{\delta_{r d}^{2}}\right)\left(\frac{2^{\frac{2 T R}{(T-\tau)}}-1}{\gamma}\right)^{2} \\
+\left(1-\mathrm{P}_{D}(\tau, k, \eta)\right) P\left(H_{1}\right) \frac{1}{2 \delta_{s d}^{2}} \\
\cdot\left(\frac{1}{\delta_{s r}^{2}}+\frac{1}{\delta_{r d}^{2}}\right)\left(\frac{\left(2^{\frac{2 T R}{(T-\tau)}}-1\right)\left(1+\gamma_{p}\right)}{\gamma}\right)^{2}
\end{aligned}
$$

The outage performance optimization problem with cooperative sensing is formulated to minimize the outage probability of cognitive relay network by using $\tau, k$ and $\eta$ as the optimization variables subject to adequate protection given to the primary user, as shown by the following:

$$
\begin{aligned}
\min _{\tau, k} \mathrm{P}_{\text {out }}(\tau, k, \eta) & \\
\text { s.t } \quad 0 & \leq \tau \leq T \\
1 & \leq k \leq M \\
\mathrm{P}_{D}(\tau, k, \eta) & \geq \overline{\mathrm{P}}_{D}
\end{aligned}
$$

where $\overline{\mathrm{P}}_{D}$ is the minimum probability of detection that the fusion center needs to protect the primary user. Given detection probability $\mathrm{P}_{D}(\tau, k, \eta)=\overline{\mathrm{P}}_{D}, A=\frac{1}{2 \delta_{s d}^{2}}\left(\frac{1}{\delta_{s r}^{2}}+\frac{1}{\delta_{r d}^{2}}\right)$ and $B=\left(1-\mathrm{P}_{D}(\tau, k, \eta)\right) P\left(H_{1}\right)$. Fixing the fusion rule threshold $k=\tilde{k}$ and the detection threshold $\eta, A$ and $B$ are constants and greater than zero. Therefore, formula (7) becomes the function with parameter $\tau$. Making the first derivative of $\mathrm{P}_{\text {out }}(\tau)$ with respect to $\tau$, we can obtain:

$$
\begin{aligned}
\nabla \mathrm{P}_{\text {out }}(\tau) & =-P\left(H_{0}\right) A\left(\frac{2^{\frac{2 T R}{(T-\tau)}}-1}{\gamma}\right)^{2} \nabla \mathrm{P}_{F}(\tau) \\
+ & P\left(H_{0}\right)\left(1-\mathrm{P}_{F}(\tau, \tilde{k}, \eta)\right) \\
& \cdot A \frac{4 T R \ln 2}{(T-\tau)^{2} \gamma^{2}} 2^{\frac{2 T R}{(T-\tau)}}\left(\frac{2^{\frac{2 T R}{(T-\tau)}}-1}{\gamma}\right) \\
+ & P\left(H_{0}\right) \nabla \mathrm{P}_{F}(\tau) \\
+ & A B \frac{4 T R \ln 2\left(1+\gamma_{p}\right)^{2}}{(T-\tau)^{2} \gamma^{2}} 2^{\frac{2 T R}{(T-\tau)}}\left(\frac{2^{\frac{2 T R}{(T-\tau)}}-1}{\gamma}\right)
\end{aligned}
$$

where $\nabla \mathrm{P}_{F}(\tau)$ can be achieved by making the first derivative of $\mathrm{P}_{F}(\tau, \tilde{k}, \eta)$ with respect to $\tau$; $\nabla \mathrm{P}_{F}(\tau)$ is expressed as:

$$
\begin{aligned}
\nabla P_{F}(\tau)= & \tilde{k}\left(\begin{array}{c}
M \\
\tilde{k}
\end{array}\right) P_{f}(\tau)^{\tilde{k}-1}\left(1-P_{f}(\tau)\right)^{M-\tilde{k}} \nabla P_{f}(\tau) \\
& +\sum_{i=\tilde{k}+1}^{M} i \frac{M !}{i !(M-i) !} P_{f}(\tau)^{i-1} \\
& \cdot\left(1-P_{f}(\tau)\right)^{M-i} \nabla P_{f}(\tau) \\
& -\sum_{i=\tilde{k}}^{M-1}(M-i) \frac{M !}{i !(M-i) !} P_{f}(\tau)^{i} \\
& \cdot\left(1-P_{f}(\tau)\right)^{M-i-1} \nabla P_{f}(\tau)
\end{aligned}
$$


Taking into account

$$
\begin{gathered}
\sum_{i=\tilde{k}+1}^{M} i \frac{M !}{i !(M-i) !} P_{f}(\tau)^{i-1}\left(1-P_{f}(\tau)\right)^{M-i} \nabla P_{f}(\tau) \\
\stackrel{m=i-1}{=} \sum_{m=\tilde{k}}^{M-1} \frac{M !}{m !(M-m-1) !} P_{f}(\tau)^{m} \\
\cdot\left(1-P_{f}(\tau)\right)^{M-m-1} \nabla P_{f}(\tau)
\end{gathered}
$$

we can obtain

$$
\begin{aligned}
\nabla P_{F}(\tau)= & \tilde{k}\left(\begin{array}{c}
M \\
\tilde{k}
\end{array}\right) P_{f}(\tau)^{\tilde{k}-1} \\
& \cdot\left(1-P_{f}(\tau)\right)^{M-\tilde{k}} \nabla P_{f}(\tau)
\end{aligned}
$$

where $\nabla \mathrm{P}_{F}(\tau)$ is given as

$$
\begin{aligned}
& \nabla P_{f}(\tau)=-\frac{1}{\sqrt{2 \pi}} \frac{\gamma_{p} \sqrt{f_{s}}}{2 \sqrt{\tau}} \\
& \cdot \exp \left(-\frac{\left(Q^{-1}\left(\overline{\mathrm{P}}_{d}^{i}\right) \sqrt{\left(2 \gamma_{p}+1\right)}+\gamma_{p} \sqrt{\tau f_{s}}\right)^{2}}{2}\right)
\end{aligned}
$$

When $\tau \rightarrow 0$, it can be deduced that $\lim _{\tau \rightarrow 0} \nabla P_{f}(\tau)=-\infty$ and $\lim _{\tau \rightarrow 0} \nabla \mathrm{P}_{F}(\tau)=-\infty$. Since $A\left(\frac{2^{\frac{2 T R}{(T-\tau)}}-1}{\gamma}\right)^{2}<1$, we have $\lim _{\tau \rightarrow 0} \nabla \mathrm{P}_{\text {out }}(\tau)=-\infty$ When $\tau \rightarrow T$, it can be deduced that $\lim _{\tau \rightarrow T} \nabla \mathrm{P}_{\text {out }}(T)=\infty$. Therefore, we can obtain that there exists an optimal sensing time to make $\nabla \mathrm{P}_{\text {out }}(\tau)=0$ among the range $0 \leq \tau \leq T$. The optimal sensing time, which can minimize the outage probability of cognitive relay network, can be obtained when the equality constraint in formula (7) is satisfied. Given the sensing time $\tau$, detection threshold $\eta$ and the number of secondary users $M$, the outage probability can be formulated as a function with respect to fusion rule threshold $k$. Letting $a=\frac{1}{2 \delta_{s d}^{2}}\left(\frac{1}{\delta_{s r}^{2}}+\frac{1}{\delta_{r d}^{2}}\right)\left(\frac{2^{\frac{2 T R}{(T-\tau)}}-1}{\gamma}\right)^{2}$ and $b=\frac{1}{2 \delta_{s d}^{2}}\left(\frac{1}{\delta_{s r}^{2}}+\frac{1}{\delta_{r d}^{2}}\right)\left(\frac{\left(2^{\frac{2 T R}{(T-\tau)}}-1\right)\left(1+\gamma_{p}\right)}{\gamma}\right)^{2}$,

$a$ and $b$ are constants and greater than zero. The outage probability optimization problem of cognitive relay network can be turned into

$$
\begin{aligned}
\mathrm{P}_{\text {out }}(k)= & \mathrm{P}_{F}(k) P\left(H_{0}\right) \\
& +P\left(H_{0}\right)\left(1-\mathrm{P}_{F}(k)\right) a \\
& +\left(1-\mathrm{P}_{D}(k)\right) P\left(H_{1}\right) b
\end{aligned}
$$

Making the first derivative of $\mathrm{P}_{\text {out }}(k)$ with respect to $k$, we can obtain

$$
\begin{aligned}
\frac{\partial \mathrm{P}_{\text {out }}(k)}{\partial k} & =\mathrm{P}_{\text {out }}(k+1)-\mathrm{P}_{\text {out }}(k) \\
& =b P\left(H_{1}\right)\left(\begin{array}{c}
M \\
k
\end{array}\right) P_{d}^{k}\left(1-P_{d}\right)^{M-k} \\
& -P\left(H_{0}\right)(1-a)\left(\begin{array}{c}
M \\
k
\end{array}\right) P_{f}^{k}\left(1-P_{f}\right)^{M-k}
\end{aligned}
$$

It is noted that $\mathrm{a}$ and $\mathrm{b}$ are positive constants, and $a<1$. When $\frac{\partial \mathrm{P}_{\text {out }}(k)}{\partial k}=0$, there exists an optimal fusion rule threshold to minimize the outage probability of cognitive relay network, and meet

$$
\begin{aligned}
b P\left(H_{1}\right) & \left(\begin{array}{c}
M \\
k
\end{array}\right) P_{d}^{k}\left(1-P_{d}\right)^{M-k} \\
& =P\left(H_{0}\right)(1-a)\left(\begin{array}{c}
M \\
k
\end{array}\right) P_{f}^{k}\left(1-P_{f}\right)^{M-k}
\end{aligned}
$$

The optimal fusion rule $k_{\text {opt }}$-out-of- $M$ fusion rule can be achieved from formula (15), which is given as

$$
k_{\text {opt }}=\left[\frac{\ln \frac{P\left(H_{0}\right)(1-a)}{b P\left(H_{1}\right)}+M \ln \left(\frac{1-P_{f}}{1-P_{d}}\right)}{\ln \left(\frac{1-P_{f}}{1-P_{d}}\right)+\ln \left(\frac{P_{d}}{P_{f}}\right)}\right]
$$

where $\lceil x\rceil$ denotes the smallest integer not less than $x$. We can find that the outage performance of cognitive relay network can be further improved by optimizing fusion rule threshold $k$. 


\section{Performance Evaluation}

In this section, computer simulation results are presented to evaluate the outage performance of cognitive relay network. We have set $\delta_{s d}^{2}=1$, $\delta_{s r}^{2}=5$ and $\delta_{r d}^{2}=5, \overline{\mathrm{P}}_{d}=0.95, P\left(H_{0}\right)=0.8$, $T=20 \mathrm{~ms}$. Assuming that the modulated signal bandwidth of primary users is $6 \mathrm{MHz}$, then the sample frequency is set to $f_{s}=6 \mathrm{MHz}$.

Figure 3 shows the comparison of analytical and simulated outage probability. We have set the signal to noise from primary user is $\gamma_{p}=-17 \mathrm{~dB}$. Figure 3(a) illustrates the outage probability for high SNR, Figure 3(b) gives the outage probability under different $\gamma_{p}$ at $\gamma=15 \mathrm{~dB}$. From Figure 3, it is observed that the analytical results match the simulated results at higher SNR region.

Figure 4 shows the outage probability versus

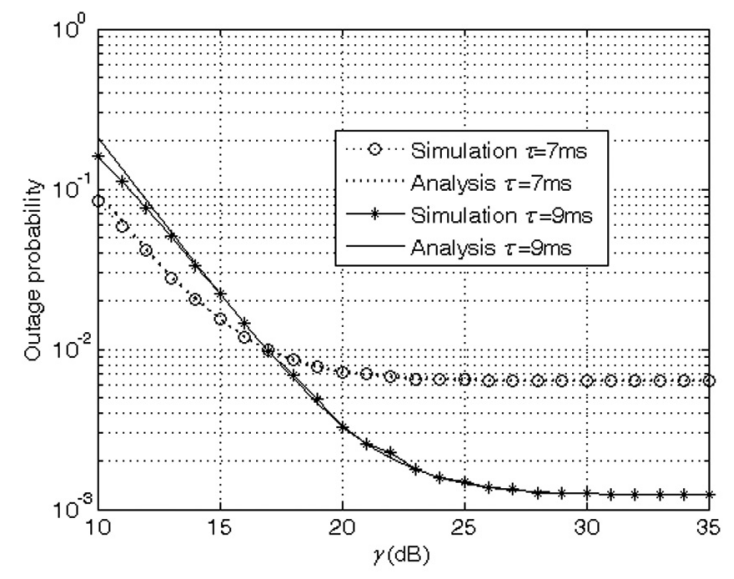

(a) Outage probability versus high SNR

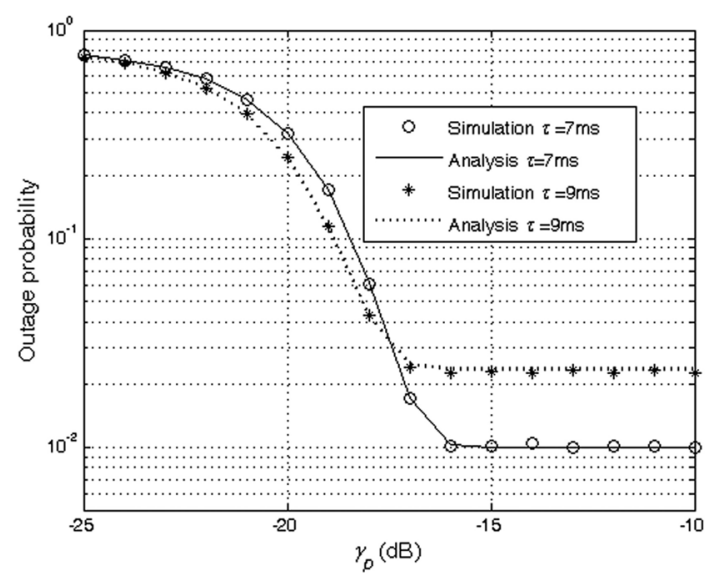

(b) Outage probability versus different $\gamma_{p}$.

Figure 3. Comparsion of analytical and simulated outage probability. fusion rule threshold $k$ under different $M$ at $\gamma_{p}=-20 \mathrm{~dB}$. It is easy to see that the outage probability decreases with the increase of $M$ at the same $k$. Furthermore, there exists an optimal $k$ for different $M$ to minimize the outage probability of cognitive relay network. Therefore, optimal fusion rule could optimize the outage performance of cognitive relay network.

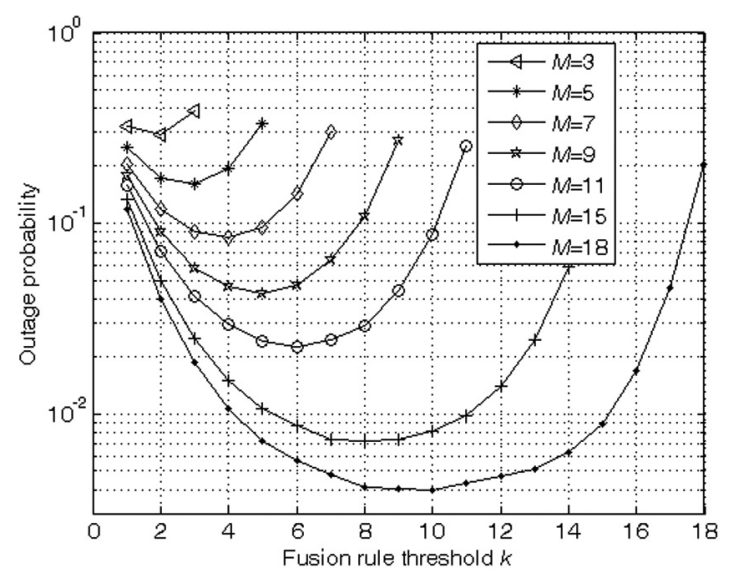

Figure 4. Outage probability versus $k$ under different $M$.

When $S N R=10 \mathrm{~dB}$ and $\tau=3 \mathrm{~ms}$, Figure $5 \mathrm{dem}$ onstrates optimal fusion rule threshold $k_{\text {opt }}$ versus $\gamma_{p}$ under different $M$, which can minimize the outage probability of cognitive relay network. It is obvious that the optimal fusion rule threshold is different with different $\gamma_{p}$.

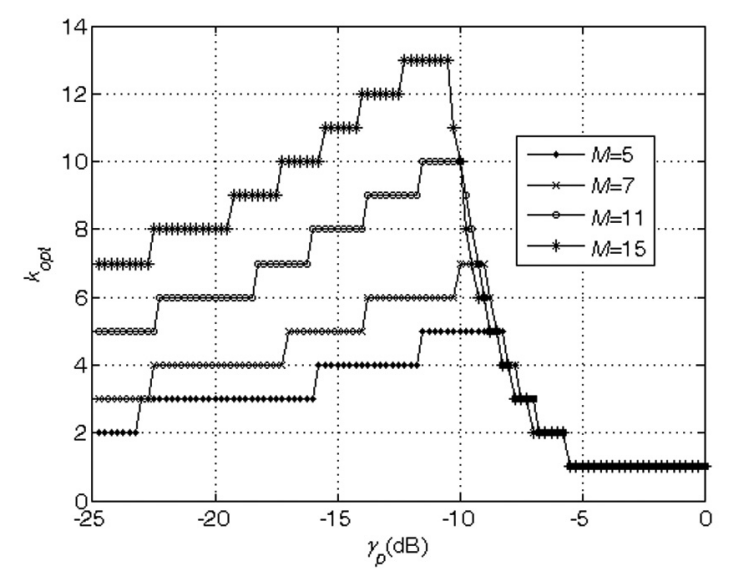

Figure 5. $k_{\text {opt }}$ versus $\gamma_{p}$ under different $M$.

Figure 6 shows the outage probability and optimal sensing time $\tau_{\text {opt }}$ versus $\gamma_{p}$ for different sensing schemes. We have set $M=5$. From Figure 6(a), we know that the outage performance based on cooperative sensing (i.e., OR rule, 


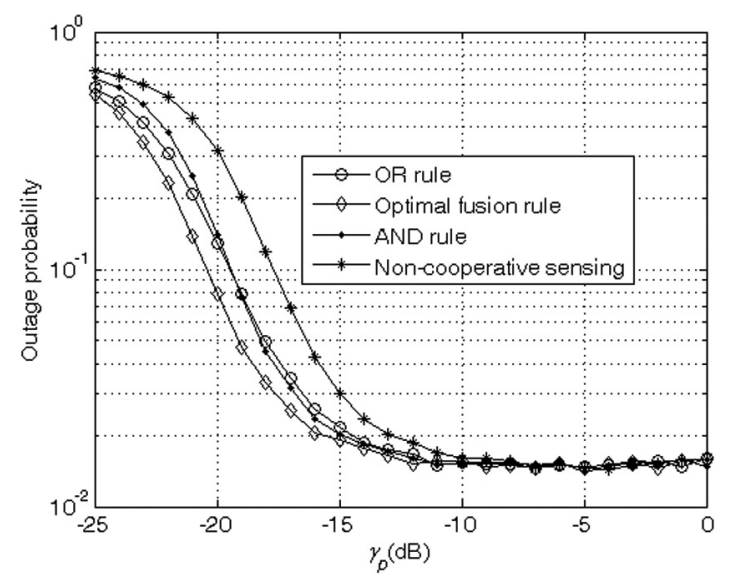

(a) Outage probability versus $\gamma_{p}$ for different sensing schemes

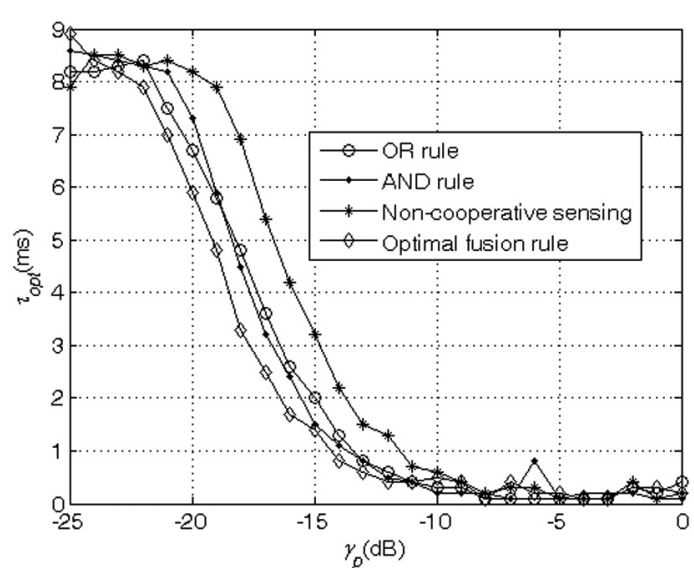

(b) $\tau_{\text {opt }}$ versus $\gamma_{p}$ for different sensing schemes

Figure 6. Performance comparison for different sensing schemes.

AND rule, Optimal fusion rule) is superior to that of non-cooperative sensing. Furthermore, the outage performance based on optimal fusion rule is the best. From Figure 6(b), it is observed that there is different optimal sensing time $\tau_{\text {opt }}$ for different $\gamma_{p}$ to minimize the outage probability of cognitive relay network. In addition, for different sensing schemes, the optimal sensing time is different. The optimal sensing time $\tau_{\text {opt }}$ based on optimal fusion rule is the smallest, then $\tau_{\text {opt }}$ based on non-cooperative sensing scheme is the biggest.

From Figure 5, we know that the optimal fusion rule is 3-out-of-5 fusion rule when $M=5$, $\gamma_{p}=-17 \mathrm{~dB}$. In Figure 7, we show the outage probability of cognitive relay network versus sensing time for different sensing schemes.

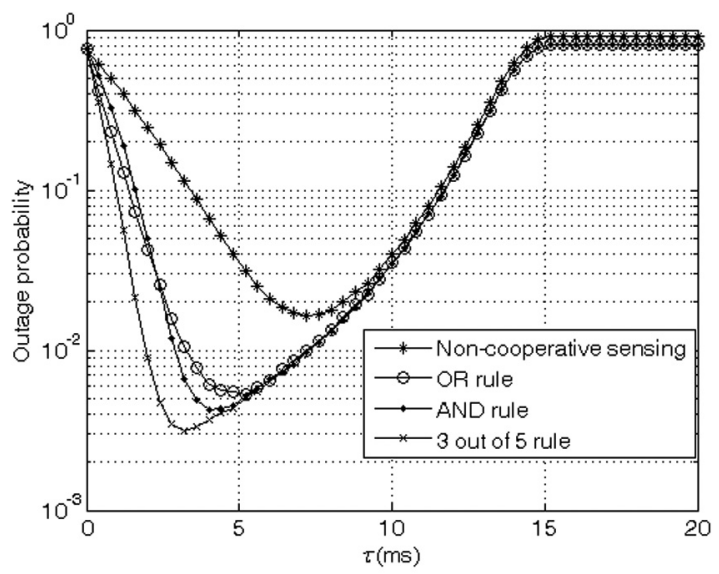

Figure 7. Outage probability comparision for different sensing schemes.
One can see that there is optimal sensing time to minimize the outage probability of cognitive relay network for different sensing schemes. Furthermore, the outage performance based on cooperative sensing schemes outperforms that of non-cooperative sensing scheme. Obviously, the outage performance based on 3-out-of-5 fusion rule is the best.

\section{Conclusion}

In this paper, we have theoretically analyzed and simulated the outage performance of cognitive relay network by considering cooperative spectrum sensing technology. Extensive simulation and numerical results show that the derived expression of outage probability matches the simulated results at high SNR region. Our idea proves that the outage performance of cognitive relay network can be significantly improved by optimally designing spectrum sensing duration and fusion rule threshold. It is noticed that when the received signal to noise ratio of primary user measured at the secondary receiver is different, there exists a different optimal fusion rule to minimize the outage probability of cognitive relay network. Last but not least, the outage performance based on cooperative sensing outperforms that of non-cooperative sensing.

\section{Acknowledgement}

This work was supported by the Foundation of Yunnan Province under grant No. 2011FB035 
and School training fund under grant No. KKZ3201403010. the National Natural Science Foundation of China (No. 61561029), the Major Project of Education Department of Yunnan Province (No. 2014Z022) and the Talent Development Project of Kunming University of Science and Technology (KKSY201403024).

\section{References}

[1] I. F. Akyildiz et al., "Cooperative spectrum sensing in cognitive radio networks: A survey", Physical Communication, vol. 4, no. 1, pp. 40-62, 2011.

http://dx.doi.org/10.1016/j.phycom.2010.12.003

[2] G. Ganesan and Y. Li, "Cooperative Spectrum Sensing in Cognitive Radio, Part I: Two User Networks", IEEE Transactions on Wireless Communications, vol. 6, no. 6, pp.2204-2213, 2007. http://dx.doi.org/10.1109/TWC.2007.05776

[3] J. Shen et al., "Maximum channel throughput via cooperative spectrum sensing in cognitive radio networks", IEEE Transactions on Wireless Communications, vol. 8, no. 10, 2009, pp.5166-5175. http://dx.doi.org/10.1109/TWC.2009.081110

[4] G. Ganesan and Y. Li, "Cooperative spectrum sensing in cognitive radio networks", in First IEEE International Symposium on New Frontiers in Dynamic Spectrum Access Networks, Nov. 2005, pp. 137-143.

http://dx.doi.org/10.1109/dyspan.2005.1542628

[5] S. Maleki et al., "Energy and throughput efficient strategies for cooperative spectrum sensing in cognitive radios", in IEEE 12th International Workshop on Signal Processing Advances in Wireless Communications, June 2011, pp.71-75. http://dx.doi.org/10.1109/SPAWC.2011.5990482

[6] Y.-J. Choi et al., "Throughput analysis of cooperative spectrum sensing in Rayleigh-faded cognitive radio systems", IET Communications, 2012, 6 (9), pp. 1104-1110.

http://dx.doi.org/10.1049/iet-com.2010.1025

[7] Y.-C. Liang et al., "Sensing-Throughput Tradeoff for Cognitive Radio Networks", IEEE Transactions on Wireless Communications, vol. 7, no. 4, pp. 1326-1337, 2008.

http://dx.doi.org/10.1109/TWC.2008.060869

[8] G. Nie et al., "Sensing-Throughput Tradeoff in Cluster-Based Cooperative Cognitive Radio Networks: A Novel Frame Structure", in IEEE 75th Vehicular Technology Conference, May 2012, pp. 1-5.

http://dx.doi.org/10.1109/vetecs.2012.6240200
[9] Y. Zou et al., "Outage Probability Analysis of Cognitive Transmissions: Impact of Spectrum Sensing Overhead", IEEE Transactions on Wireless Communications, vol.9, no. 8, pp. 2676-2688, 2010.

http://dx.doi.org/10.1109/TWC.2010.061710.100108

[10] F. A. Khan et al., "Outage performance of cognitive radio wireless network with secondary relaying", in 2012 International Conference on Computer Systems and Industrial Informatics (ICCSII), 2012.

http://dx.doi.org/10.1109/ICCSII.2012.6454571

[11] Z. Xing et al., "Outage Performance Study of Cognitive Relay Networks with Imperfect Channel Knowledge", IEEE Communications Letters, vol. 17, no. 1, pp.27-30, 2013.

http://dx.doi.org/10.1109/LCOMM.2012.112812.121371

[12] X. Zhang et al., "On the Study of Outage Performance for Cognitive Relay Networks (CRN) with the Nth Best-Relay Selection in Rayleigh-fading Channels", IEEE Wireless Communications Letters, vol. 2, pp. 110-113, 2013.

http://dx.doi.org/10.1109/WCL.2012.120312.120701

[13] Z. Feng et al., "Outage performance of relay-assisted primary and secondary transmissions in cognitive relay networks", EURASIP Journal on Wireless Communications and Networking, vol. 60, December 2014.

http://dx.doi.org/10.1186/1687-1499-2014-60

[14] J. Tao Jing et al., "Achievable transmission capacity of cognitive radio networks with cooperative relaying", EURASIP Journal on Wireless Communications and Networking, pp. 94, December 2015.

http://dx.doi.org/10.1186/s13638-015-0311-8

[15] Y. Zhao et al., "Improving amplify-and-forward relay networks: optimal power allocation versus selection", IEEE Transactions on Wireless Communications, vol. 6, no. 8, pp. 3114-3123, 2007.

[16] J. Laneman et al., "Cooperative diversity in wireless networks: efficient protocols and outage behavior", IEEE Trans Inf Theory, vol. 50, pp. 3062-3080, 2004.

http://dx.doi.org/10.1109/TIT.2004.838089

Received: March, 2015 Revised: December, 2015

Accepted: January, 2016

Contact addresses:

Yaolian Song

Faculty of Information Engineering and Automation

Kunming

University of Science and Technology

Chenggong Campus

Kunming University of Science and Technology Kunming

Yunnan Province

Postcode: 650504

China

e-mail: sy1719@163.com 
YuHao Li

Faculty of Information Engineering and Automation

Kunming

University of Science and Technology

Chenggong Campus

Kunming University of Science and Technology Kunming

Yunnan Province

Postcode: 650504

China

e-mail: 534693041@qq.com

Shujuan Wang

Faculty of Information Engineering and Automation

Kunming

University of Science and Technology

Chenggong Campus

Kunming University of Science and Technology Kunming

Yunnan Province

Postcode: 650504

China

e-mail: shujuanwang0703@126.com

Ya Zhang

Faculty of Information Engineering and Automation

Kunming

University of Science and Technology

Chenggong Campus

Kunming University of Science and Technology Kunming

Yunnan Province

Postcode: 650504

China

e-mail: 1050336280@qq.com
YaOLIAN Song received her Ph.D. degree in Communication and Information Systems from Nanjing University of Posts and Telecommunications. She is currently an Associate Professor at the Faculty of Information Engineering and Automation, Kunming University of Science and Technology, Yunnan, China. Her research interests include wireless communication, cooperative communication and cognitive radio.

YuHao Li is a Master graduate student of professional electronic and communication systems at Kunming University of Science and Technology, School of the Faculty of Information Engineering and Automation.

ShuJUAN WANG received the B.S. degree in Biomedical Engineering from Beijing Jiaotong University, Beijing, China, in 2007 and the Ph.D. degree in Signal and Information Processing from the same University, in 2013. From 2010 to 2011, she was a visiting scholar at The Fu Foundation School of Engineering and Applied Science, Columbia University, NY, USA.

She is currently an Assistant Professor at the Faculty of Information Engineering and Automation, Kunming University of Science and Technology, Yunnan, China. Her research interests include data dissemination, vehicular communications, network coding and wireless communications.

YA ZHANG is a Master graduate student of professional signal and information processing at Kunming University of Science and Technology, School of the Faculty of Information Engineering and Automation. 